\title{
Automatic Obligation Generation and Monitor System for Privacy Policy to DBMS
}

\author{
Mary Treesa Thomas \\ PG Scholar, \\ Department of CSE(PG), \\ Sri Ramakrishna Engineering \\ College, \\ Coimbatore.
}

\author{
Kanagaraj R. \\ Assistant Professor (Sr.Gr), \\ Department of Software \\ Engineering(PG), \\ Sri Ramakrishna Engineering \\ College, \\ Coimbatore.
}

\author{
Lakshmi Vidyadharan \\ PG Scholar, \\ Department of CSE(PG), \\ Sri Ramakrishna Engineering \\ College, \\ Coimbatore.
}

\begin{abstract}
Data stored in the database includes personnel and sensitive data. Privacy policies in the database management system can control collection, access and disclosure of data. Policies are used to specify obligation. Obligation is certain actions or condition which has to be satisfied for accessing data. The main objective this project is to enable a monitor for implementing privacy policies which specifies obligations. Based on the obligations monitor can control the SQL code execution. The approach is automated, systematic and can work with most of the relational DBMS.
\end{abstract}

\section{Keywords}

Obligations, Privacy policies, Relational database management system

\section{INTRODUCTION}

Network Security contains rules and regulations which are guided by network administrator who controls the authorization of access to data in a network. Users will be assigned with user ID and password to access the information. This will prevent unauthorized access, misuse, modification, or denial of a computer network and its resources.

Database security ensures confidentiality, integrity and availability properties of the stored data. Several technical, administrative and physical controls are applied. Unauthorized access, malware infections, overloads, physical damage to the servers, design flaws and data loss are some security risks of database systems. Several controlling measures in database are access control, auditing, authentication, encryption, integrity controls, backups etc. Firewalls and intrusion detection systems are network security measures to secure database from hacking. Many organizations have their own standard measures for database security. This provides additional security based on their obligations along with the general security measures. The administrative and management functions of database security involve administration and reporting of user access rights, log management and database replication/synchronization and backups. Hence database security is an important thing in this era in order to protect the sensitive data.

\subsection{Overview of the Project}

Policies are used to specify obligation. Obligation is certain actions or condition which has to be satisfied for accessing data. There are no proper specification tools in access control system. System administrators are able to change the policies of their access control system that is they can modify obligations. This programming activity is ad-hoc. It is hard to find which obligations are handled by the system. Also this programming activity is erroneous since it does not have proper specification language and there are no frameworks for measuring the correctness of system. This ad-hoc programming activity is not flexible. Slight changes in the policies results in consistency problem. XACML and EPAL are the two standard languages to support specification of policies and obligations, but this support is partial only. Several researches have been done to implement strategy that support specification of obligations and monitoring system which can adapt with relational database management system. Such programs should be able to block or modify SQL command execution in order to comply with the obligations created for the data.

\subsection{Principles of Data security}

Data privacy, also called information privacy, is the aspect of information technology (IT) that deals with the ability an organization or individual has to determine what data in a computer system can be shared with third parties

\subsubsection{Obligations}

Obligations control the operations performed in database and analyze its current state. Also, obligations are used to provide a monitoring system that 1) monitors the activities of DBMS, and 2) analyze current state of accessed database and command execution history. Privacy policies are used to define obligations which can control the access and collection of data in the database. Privacy policies use certain specific terms which are users or roles, actions, purposes and conditions. All these terms are used to enforce privacy policies in the relational DBMS. The approaches of this work is to 1) define privacy policies and the obligations2) enforcement of policies in relational DBMS. The existing system, Purpose and Role Based access control which is an enforcement monitor. It contains PuRBAC [1] policies which tracks the SQL query execution. It ensures that the purpose of data accessing should comply with purpose for which data are collected and only authorized users who belong to an authorized role can only requests query execution.

\subsection{Enforcement of Privacy}

The proposed work defines an extended PuRBAC (ePuRBAC), which is a monitoring system in addition to the PuRBAC for enforcing obligations in the relational DBMS. Modeling and analysis of privacy aware systems (MAPaS) [2], [3], which is used for the development of privacy aware system. MAPaS supports the privacy policy specification and definitions of enforcement mechanisms. Model driven engineering technologies and aspect oriented development approach are used to support the development of ePuRBAC. The main advantage 
of the proposed system is there is no need of programming activity to the system administrators; they only need to do the privacy policy specifications and some options for interaction and configurations. ePuRBAC unifies all aspects of the privacy policies such as obligations, purpose of access, roles, authorizations and conditions. ePuRBAC avoids ad-hoc programming because it is generated automatically. So its development takes less time. Enforcement of recursive obligations that is the obligations that monitors the current state of the system and checks the development of the in specific time intervals are supported by the proposed work. Hence ePuRBAC is a strong obligation model. The execution of queries is based on the temporal patterns of the system. ePuRBAC can work within almost all relational DBMSs. Earlier works does not have frameworks for developing privacy enforcement monitor which can be used in relational DBMSs with such features.

\subsection{Need for the Study}

Enforcement monitor which handles privacy policies contains obligations in different policy specification language. Such a monitor is derived from the same set of policies that must be enforced, and regulates the execution of SQL code based on the satisfaction of a variety of obligation types. A possible adaptation strategy consists in implementing adapter modules that interact with the obligation enforcement framework and the DBMS. Such modules should monitor, block or modify the execution of SQL commands, in such a way that the access complies with the obligations defined for the accessed data. Obligations control the operations performed in database and analyze its current state. Also, obligations are used to provide a monitoring system that 1 ) monitors the activities of DBMS, and 2) analyze current state of accessed database and command execution history. Privacy policies are used to define obligations which can control the access and collection of data in the database. Privacy policies use certain specific terms which are users or roles, actions, purposes and conditions. All these elements are needed in this.

\section{PROPOSED SYSTEM}

Obligations are the conditions that must be satisfied or the actions that must be performed before or after the execution of SQL queries. Policies are used to specify obligation. There are no proper specification tools in access control system. Privacy policies are specified by a modeling language called PaML, which is a UML profile. PaML specifications are then enforced with PuRBAC. System administrators are able to change the policies of their access control system that is they can modify obligations. This programming activity is ad-hoc. It is hard to find which obligations are handled by the system. Also this programming activity is erroneous since it does not have proper specification language and there are no frameworks for measuring the correctness of system. This ad-hoc programming activity is not flexible. Slight changes in the policies results in consistency problem. The concept of obligations in access control system has emerged recently.

\subsection{Problem Objective}

The approaches of this work is to 1) define privacy policies and the obligations2) enforcement of policies in relational DBMS. The existing system, Purpose and Role Based access control which is an enforcement monitor. It contains PuRBAC policies which tracks the SQL query execution. It ensures that the purpose of data accessing should comply with purpose for which data are collected and only authorized users who belongs to an authorized role can only requests query execution. The system needs to define the PaML model first. It is then enforced by PuRBAC. PaML model contains 1) purposes and roles 2) intended purpose and its data association 3) authorized users 4) access purpose authorization. The proposed work defines an extended PuRBAC (ePuRBAC), which is a monitoring system in addition to the PuRBAC for enforcing obligations in the relational DBMS. Modeling and analysis of privacy aware systems(MAPaS), which is used for the development of privacy aware system. MAPaS supports the privacy policy specification and definitions of enforcement mechanisms. Model driven engineering technologies [5] and aspect oriented development approach [4] are used to support the development of ePuRBAC. The main advantage of the proposed system is there is no need of programming activity to the system administrators, they only need to do the privacy policy specifications and some options for interaction and configurations. ePuRBAC unifies all aspects of the privacy policies such as obligations, purpose of access, roles, authorizations and conditions[7], [8]. ePuRBAC avoids ad-hoc programming because it is generated automatically. So its development takes less time. Enforcement of recursive obligations that is the obligations that monitors the current state of the system and checks the development of the in specific time intervals are supported by the proposed work. Hence ePuRBAC is a strong obligation model. The execution of queries is based on the temporal patterns [9] of the system. ePuRBAC can work within almost all relational DBMSs. When an action is invoked obligation can define the state of data [10] in database. It can also specify what all actions can be executed at a given time. There are pre-obligations [11], [12] which is the conditions that must be satisfied before the execution of an action and postobligations which are the conditions that must be satisfied after the execution of an action. Earlier works does not have frameworks for developing privacy enforcement monitor which can be used in relational DBMSs with such features.

\subsection{Architecture Diagram}

User who wants to access the database should belong to an authorized role. Their purpose for accessing database should be authorised.User who requests the activation of a given action (whose specification is provided in a PaML model by invoking operation enable of activator. If there exists an access purpose authorization which is valid for the user, activate action is invoked. Once an execution request has been received, action checks whether the preconditions to the execution are satisfied. When a user requests an action, it is verified by applying obligations and blocking those actions which violates the defined policies that is ePuRBAC, a monitor that performs runtime enforcement of privacy policies that include obligations within relational DBMSs.

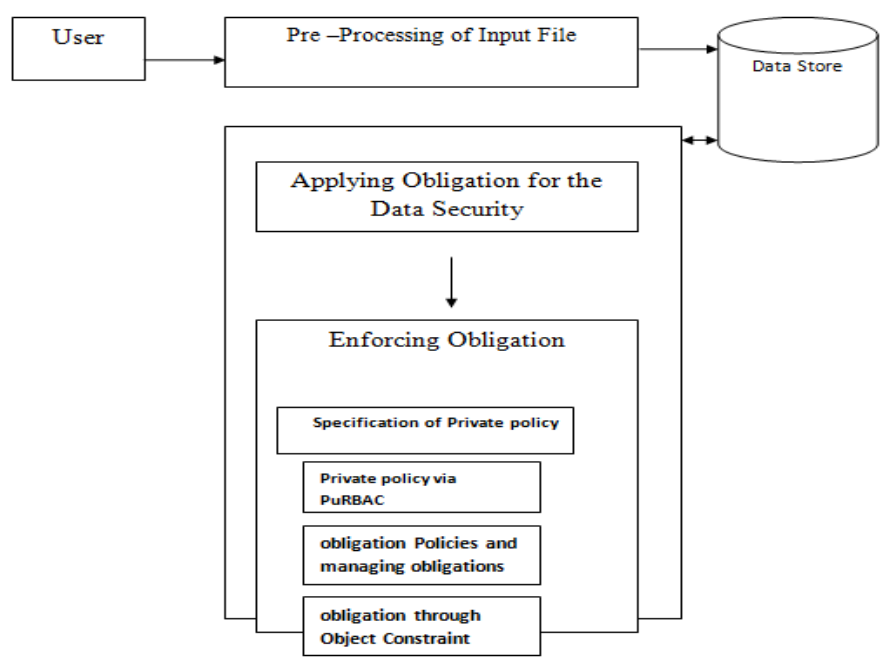

Fig 2.1. Architecture of Proposed System 


\subsection{Advantages}

- Automatically generated obligations for the privacy policies.

- Verifies the system obligation periodically and updates in specific time intervals.

- Error prone rate is low.

- System is flexible.

\section{RELATED WORK}

Colombo P and Ferrari E, (2012)“Towards a Framework to Handle Privacy Since the Early Phases of the Development: Strategies and Open Challenges" Although almost any software application processes personal data, effective development frameworks that properly handle privacy are still missing. This work makes a step to fill this void. This literature investigates requirements and development strategies of a privacy-preserving development framework that deals with privacy since the early phases of the development.

Katt, Zhang, R. Breu, M. Hafner, and J.-P. Seifert, (2008)“A General Obligation Model and Continuity: Enhanced Policy Enforcement Engine for Usage Control" The usage control model (UCON) has been proposed to augment traditional access control models by integrating authorizations, obligations, and conditions and providing the properties of decision continuity and attribute mutability. Several recent works have applied UCON to support security requirements in different computing environments such as resource sharing in collaborative computing systems and data control in remote platforms. In this Literature proposed identify two individual but interrelated problems of the original UCON model and recent implementations: oversimplifying the concept of usage session of the model, and the lack of comprehensive ongoing enforcement mechanism of implementations. Proposed extend the core UCON model with continuous usage sessions thus extensively augment the expressiveness of obligations in UCON, and then propose a general, continuity-enhanced and configurable usage control enforcement engine.

Ni.Q, Bertino, and Lobo,(2008)“An Obligation Model Bridging Access Control Policies and Privacy Policies" This paper presents a novel obligation model for the Core Privacy-aware Role Based Access Control (P-RBAC), and discuss some design issues in detail. Pre-obligations, post-obligations, conditional obligations, and repeating obligations are supported by the obligation model. Interaction between permissions and obligations is discussed, and efficient algorithms are provided to detect undesired effects.

\section{EXPERIMENTAL ANALYSIS}

The performance of the proposed system has been evaluated and proved its efficiency. Chart showing the presentation of execution time against affected values versus sequence values and rules against the records are given.

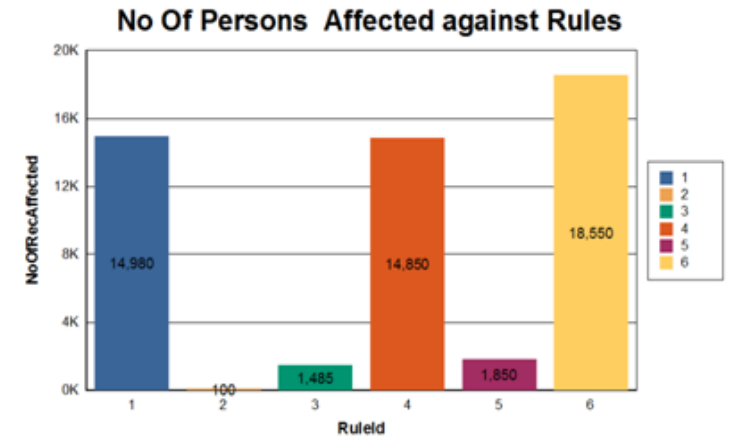

Figure 4.1 Charts Showing the Rules against the Records

\subsection{Performance Evaluation of the Rules against the Records.}

Figure 4.1. Explains an approach to the definition of ePuRBAC, a monitor that performs runtime enforcement of privacy policies that include obligations within relational DBMSs. The automated approach that generates ePuRBAC uses principles and technologies of model driven engineering and aspect oriented programming, without requiring programming activities. The delays are not due to memory management of numerous threads executed in parallel. Indeed, in the current approach a thread that handles the execution of actions is activated for every specified temporal pattern. Therefore, if a temporal specification involves multiple patterns, multiple threads are executed at the same time. Whenever a thread completes its execution the memory handled by the thread should be freed.

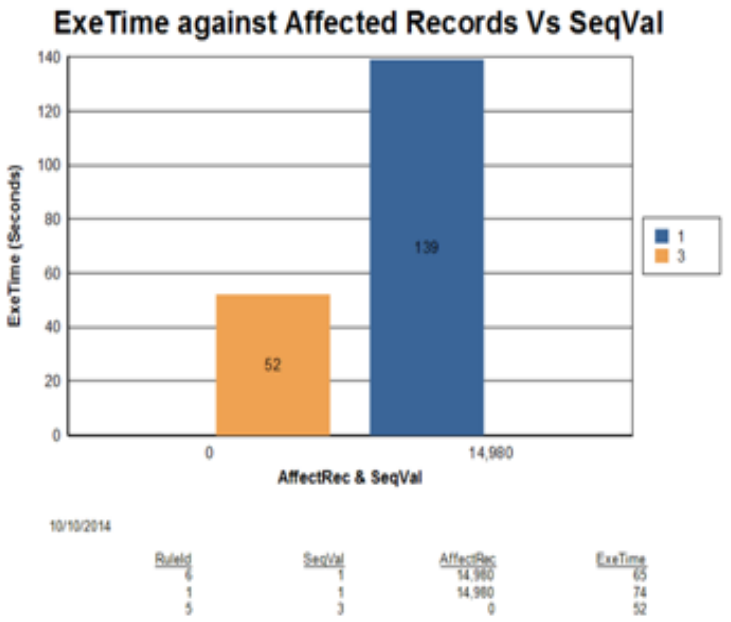

Figure 4.2: Chart showing Execution time against the records

\subsection{Performance evaluation of the execution time against the records.}

The scheduling of the activities of this tool is non deterministic, and as a consequence, unexpected delays can be introduced and can affect the scheduling of other activities in the system. To address this issue, this work is considering modifying the approach in such a way that within ePuRBAC a unique thread is generated for each composite temporal specification. By diminishing the number of threads that are concurrently executed this work optimize the memory consumption, allowing the parallel management of a bigger number of actions and obligations. This choice should reduce the number of non deterministic delays introduced by the garbage collector. 
However, since a real-time virtual machine uses a different memory model and thread management system, this choice would require to significantly modifying the ePuRBAC code.

\section{CONCLUSION}

Automatic obligation generation and monitor system for privacy policy to DBMS, has been presented and implemented an approach to the definition of ePuRBAC. ePuRBAC is a monitoring system which can automate the privacy policy enforcement which includes the obligations which can be applied within the relational DBMS. This run time enforcement is implemented with the help of model driven engineering and aspect oriented programming. This approach does not require any programming activities.

\subsection{Future Enhancement}

As future work, System has analyzed to cooperate with companies and public institutions with the aim to thoroughly analyze ePuRBAC performances through case studies. The methods specify the privacy policies introduced by several privacy regulations and privacy laws and to test the derived monitor on different DBMSs. Future plan is also to extend the obligation language to the support of an ongoing obligation.

\section{ACKNOWLEDGMENTS}

First and foremost I place this project work on the feet of GOD ALMIGHTY who is the power of strength in each step of progress towards the successful completion of my project. I am highly indebted to Dr.N.R.Alamelu, M.E., Ph.D. Principal, Sri Ramakrishna Engineering College, Coimbatore for providing the required facilities and support to carry out this project work. I extend my heartfelt thanks to Dr.A.Grace Selvarani, M.E., Ph.D. Professor and Head, Department of Computer Science and Engineering (PG), who had been a source of inspiration and for her timely guidance in the conduct of this project work. I am grateful to Mr.R.Kanagaraj, assistant professor, Department of Software Engineering (PG), for her able guidance and useful suggestions which helped me in completing the project work in time. Finally, I would like to express my heartfelt thanks to my beloved parents for their blessings, my friends for their help and wishes for the successful completion of this project.

\section{REFERENCES}

[1] Bettini.C, Jajodia.S,Wang, and Wijesekera,(2013) "Provisions and Obligations in Policy Rule Management," J. Network and Systems Management, vol. 11, no. 3, pp. 351-372.
[2] Byun and N. Li.N,(2008) "Purpose Based Access Control for Privacy Protection in Relational Database Systems," The Int'l J. Very Large Data Bases, vol. 17, no. 4, pp. 603619.

[3] Colombo and Ferrari.E, "Enforcement of Purpose Based Access Control within Relational Database Management Systems," IEEE Transaction on Knowledge and Data Eng. (IEEE TKDE), to appear.

[4] Colombo.Pand Ferrari.E, (2012)“"Towards a Framework to Handle Privacy Since the Early Phases of the Development: Strategies and Open Challenges," Proc. IEEE Sixth Int'l Conf. Digital Ecosystems Technologies (DEST).

[5] Colombo and Ferrari,(2012) "Towards a Modeling and Analysis Framework for Privacy-Aware Systems," Proc. Int'l Conf. Privacy, Security, Risk and Trust and Int'l Conf. Social Computing (PASSAT).

[6] France and Rumpe,(2007) "Model-Driven Development of Complex Software: A Research Roadmap," Proc. Future of Software Eng. (FOSE).

[7] Gama.P, C. Ribeiro, and P. Ferreira, (2006)"Heimdhal: A History-Based Policy Engine for Grids," Proc. IEEE Sixth Int'l Symp. Cluster Computing and the Grid (CCGRID).

[8] Hilty. M,Basin, and A. Pretschner,(2005) "On Obligations," Proc. European Symp. Research in Computer Security (ESORICS '05), pp. 98-117.

[9] Irwin.K, T. Yu, and W.H. Wins borough,(2006) "On the Modeling and Analysis of Obligations," Proc. 13th ACM Conf. Computer and Comm. Security (CCS).

[10] Jafari.M, P. Fong, R. Safavi Naini, and K. Barker,(2013) A Framework for Expressing and Enforcing Purpose-Based Privacy Policies.

[11] Katt, Zhang, R. Breu, M. Hafner, and J.-P. Seifert, (2008)“A General Obligation Model and Continuity: Enhanced Policy Enforcement Engine for Usage Control," Proc. 13th ACM Symp. Access Control Models and Technologies (SACMAT).

[12] Kiczales, Lamping, Mendhekar, Maeda, Lopes.J.,Loingtier, and Irwin,(1997) "Aspect-Oriented Programming," Proc. European Conf. Object-Oriented Programming (ECOOP '97), pp. 220- 242. 